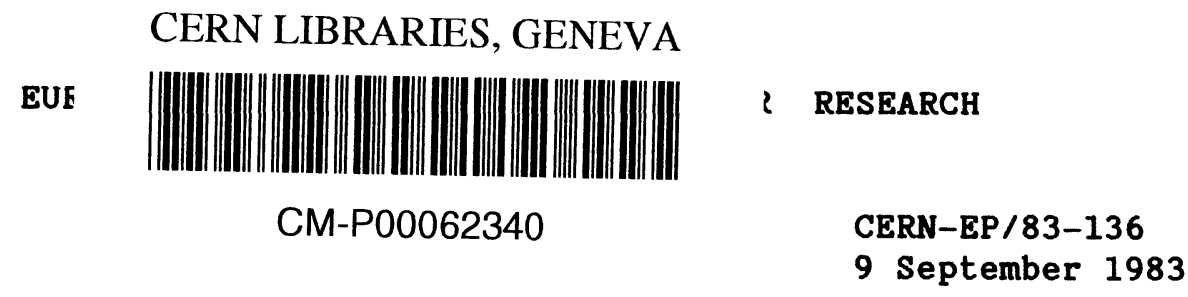

\title{
ELECTROMAGNETIC CALORIMETRY USING SCINTILLATING PLASTIC FIBRES
}

\author{
H. Burmeister, P. Sonderegger \\ CERN, Geneva, Switzerland \\ J.M. Gago, A. Maio, M. Pimenta \\ INIC, Lisbon, Portugal \\ D. Perrin \\ University of Neuchâtel, Switzerland \\ J.-C. Thévenin \\ STIPE, Saclay, Gif-sur-Yvette
}

(Presented by D. Perrin)

\section{ABSTRACT}

When used in lead-scintillator sandwich calorimetry, scintillating optical fibres offer a new way to achieve fine grain sampling and hereby to improve the energy and spatial resolution of a technique still unbeaten for timing and compactness. A small calorimeter element made of planes of $0.9 \mathrm{~mm}$ diameter fibres alternating with $\mathrm{Pb}$ plates has been built and tested in an electron beam. An energy resolution of $\sigma_{E} / \sqrt{E} \simeq 9.8 \%$ and $7.8 \%$ was obtained with $1 \mathrm{~mm}$ and $0.5 \mathrm{~mm} \mathrm{~Pb}$ plates, respectively. The parameters of the fibres have been measured. Calculations done with a refined version of the EGS code reproduce these results and suggest improved $\mathrm{Pb}$-fibre calorimeters rivalling with $\mathrm{Pb}$ glass in energy resolution. 


\section{INTRODUCTION}

The conventional lead-scintillator sandwich structure excels in compactness, in timing accuracy, and, provided it is adequately segmented, in space and two-track resolution. Its energy resolution $\sigma_{E}$ is however dominated by the sampling error and therefore limited by the difficulties and costs involved in producing very many and very thin scintillator slabs with good light transmission.

Two of us suggested to overcome this limitation by using scintillating optical fibres ${ }^{1}$, which have a good light transmission, which can be made very thin at reasonable cost so as to achieve very fine grain sampling, and whose ends can be joined flexibly to the photon converters.

We describe hereafter the structure and performance of a calorimeter element made of layers of fibres of $0.9 \mathrm{~mm}$ diameter ${ }^{2}$ alternating with $\mathrm{Pb}$ plates which was tested in an electron beam normal to the plates.

Following a suggestion by $D$. Treille that the fibres can also run almost parallel to the beam ${ }^{3}$ ), another group has built and tested a prototype meant for a cell-type calorimeter ${ }^{4}$. We evaluate, at the end of this paper, the potential of calorimeters of both types made of very thin fibres.

\section{THE SCINTILLATOR FIBRES}

Following the pioneering work of S.R. Borenstein et a1. ${ }^{5}$, scintillating optical fibres made of coated polystyrene were developed in Saclay ${ }^{2}$. Their light output was measured using a ${ }^{90} \mathrm{Sr}$ source and a carefully collimated telescope arrangement. An electron produced five photoelectrons per $\mathrm{mm}$ at $20 \mathrm{~cm}$ from the photocathode, and three at $80 \mathrm{~cm}$. A fibre of different doping and almost double light output is available. Both fibres have attenuation lengths in excess of $3 \mathrm{~m}$ beyond the first metre.

The fibres were found to be mechanically robust provided a minimal bending diameter not smaller than 100 times the fibre diameter was 
respected. Optical transmission is good within the same bending limit. Ihe fibres can be annealed, and their profile reshaped. The resistance to radiation is that of polystyrene. From $10^{5}$ rad delivered by a nuclear reactor within two hours a fibre turned yellow. However another fibre was unaffected by $4 \times 10^{4}$ rad delivered over four weeks at the ISR.

\section{THE CALORIMETER}

The test calorimeter consists of three modules of $5.4 \times 5.4 \mathrm{~cm}^{2}$ fiducial area and $11 \mathrm{~cm}$ depth each. A module contains $50 \mathrm{~Pb}$ plates of either $1 \mathrm{~mm}$ or $0.5 \mathrm{~mm}$ thickness, followed by 50 layers of 60 fibres of 0.9 $\mathrm{mm}$ diameter and of alternating perpendicular orientation (Fig. 1 , see also Fig. 1 of Ref. 2). In order to permit shower profile studies, each fibre layer was divided into six strips of ten fibres, and the corresponding strips of the 25 layers of the same orientation within a module were bundled together and read out by their own photomultiplier. We used 36 PM2973, six per module and per orientation. Cylindrical plexiglas mixers spread the light of each fibre over the photocathode area.

Two versions of the calorimeter were used: the version called hereafter " $1 \mathrm{~mm}$ " with $1 \mathrm{~mm} \mathrm{~Pb}$ plates throughout totalling 26.3 radiation lengths, and the "0.5 mm" version with two modules with $0.5 \mathrm{~mm} \mathrm{~Pb}$ plates followed by a module with $1 \mathrm{~mm}$ plates, totalling $18.4 \mathrm{~L}_{\mathrm{rad}}$.

\section{TEST RUNS AND RESULTS}

The tests were done in a negative beam at the CERN PS, whose momentum could be varied between 0.05 and $1 \mathrm{GeV} / \mathrm{c}$. The momentum spread was small. The integrated pulse heights of the 36 photomultipliers were recorded on magnetic tape event by event. The calibration was done periodically using several $10^{4}$ muons spread over the whole counter and identified by their range and by a threshold Cerenkov counter which rejected pions. On 1 y muons traversing all three modules at small angles were considered. The sum of the pulse heights recorded for each module and orientation (one or two photomultipliers for a given $\mu$ ) was equated to the computed average 
energy loss of $3.6 \mathrm{MeV}$ in 25 fibre layers, slightly corrected for the presence of the $\mathrm{Pb}$ plates. The calibration constants obtained gave energy losses uniform over the entire detector area, and varied little with time.

Electrons were identified by the threshold Cerenkov counter or, at the lowest momenta with the Cerenkov counter removed to avoid bremstrahlung losses, by time of flight. They were focussed on the centre of the calorimeter and their impact was determined in $1 \times 1 \mathrm{~mm}^{2}$ bins by a hodoscope. The integrated pulse heights of the 36 photomultipliers were converted off-line to energies using the muon calibration constants. Their sum gave the total energy deposited in the scintillators, Esc Figure 2 shows some electron and muon spectra. For each run of a few $10^{3}$ electron showers the average value $\left\langle\mathrm{E}_{\mathrm{sc}}\right\rangle$ and $\mathrm{its} \mathrm{r} . \mathrm{m} . \mathrm{s}$. fluctuation $\sigma\left(E_{s c}\right)$ were computed. Figure 3 shows, for various electron energies $E$ and for the two calorimeter versions, the ratios $<E s c / E$ and $\sigma_{E} / \sqrt{ }$, where $\sigma_{E}=$ $=E \times \sigma\left(E_{s c}\right) / E_{s c}$. In accordance with shower theory, both ratios are found to be roughly independent of energy. We find, between 0.2 and 1 $\mathrm{GeV},\left\langle\mathrm{E}_{\mathrm{sc}}>/ \mathrm{E} \simeq 0.127(0.087)\right.$ and $\sigma_{E} / \sqrt{E}=7.8 \%(9.8 \%)$ for the "0.5 mm" ("1 mm") version, with an error $\delta \sigma_{E} / \sqrt{E}=0.2 \%(0.4 \%)$ estimated from the data. The spatial resolution as derived from the centre of gravity of the shower is $\sigma_{y}=2.7 \mathrm{~mm}(3.1 \mathrm{~mm})$ at $1 \mathrm{GeV}$, and scales also as $1 / \sqrt{ }$.

Spectra of a light emitting diode facing individual photomultipliers were also analysed. The number of 21 photoelectrons per MeV deduced from the width of the observed charge distribution was consistent with the photoelectron yield measured previously for a single fibre. Electrons of $1 \mathrm{GeV}$ produce 2600 (1800) photoelectrons in the "0.5 mm" ("1 mm") version.

5. SHOWER SIMULATION FOR EXISTING AND SPECULATIVE SANDWICH STRUCTURES

Electron showers in the two calorimeter configurations were simulated by the EGS code ${ }^{6)}$ at 0.2 and $1 \mathrm{GeV}$ with 1000 and 250 showers per case, 
for flat scintillators of the same mass as the fibre layers and disregarding photoelectron statistics. The cut-off energies $(0.2 \mathrm{MeV}$ for photons, 0.5-1 MeV for electrons) were such that their variation had little effect on the results. The fluctuation $\sigma_{c}$ due to the cylindrical shape of the fibres, and the photoelectron fluctuation o were computed separately, to obtain the total r.m.s. error as $\sigma_{E}^{2}=$ $=\sigma_{E G S}^{2}+\sigma_{c}^{2}+\sigma_{p e}^{2}$.

As Table 1 and Fig. 4 show, agreement is good for the energy resolution, and fair for the energy deposited, with the simulation result lower than experiment in the first module, for all in both configurations and momenta. Part of the disagreement may be due to the slimness and complexity of the calorimeter, with incomplete overlap between fibres and lead plates, which could not be rigorously simulated. Predictions for laterally extended " $1 \mathrm{~mm}$ " and "0.5 mm" versions are also shown; the latter would achieve $\sigma_{E} / \sqrt{E}=6.9 \%$.

Figure 4 also covers the Geneva-Milan-Neuchâtel calorimeter ${ }^{7 \text { ) }}$ ( $5 \mathrm{~mm} \mathrm{~Pb}$ plates and 1iquid scintillator in teflon tubes) and the more dilute $\left(L_{r a d}=33 \mathrm{~mm}\right.$ ) ARGUS detector $\left.{ }^{8}\right)$ with its excellent energy resolution.

Since the sampling fluctuations are still the main source of error, we then contemplate a further bold reduction of sampling thickness. With $200 \mu$ fibres of improved light output and $100 \mu \mathrm{Pb}$ foils, the simulation foresees $\sigma_{E} / \sqrt{ } E=4.2 \%$, still at normal incidence, and $4.7 \%$ with the particles impinging almost parallel to the fibres, as in a cellular calorimeter ${ }^{4}$. The dependence on incidence angle is smaller than with the coarser sampling of Ref. 2. A fusther improvement is expected, again at constant overall density, with the same fibres embedded in $\mathrm{Pb}$ in a lattice configuration.

Very thin fibres can be produced at a cost per unit mass not much higher than the $1 \mathrm{~mm}$ fibre. We hope to demonstrate in the near future the technical and economical feasibility of fast and compact electromagnetic calorimeters of good energy resolution. 


\section{REFERENCES}

1) D. Perrin and P. Sonderegger, Electromagnetic calorimetry with scintillating optical fibres, CERN OM/SPS/81-7 (May 1981).

2) L.R. Allemand et al., Optical scintillating fibres for particle detectors, Proc. 2nd Pisa Meeting on Advanced Detectors (1983).

3) DELPHI Photodiode Calorimetry Group, DRLPHI Note 83-45.

4) H. Blumenfeld et al., An electromagnetic calorimeter prototype module using scintillating fibres immersed in a Pi-Pb alloy, CEA report, DPhPE 83-10.

5) S.R. Borenstein and R.C. Strand, Fine-grained hodoscopes based on scintillating optical fibres, Proc. 1981 ISABELLE Workshop, BNL-51443 (1981).

6) R.L. Ford and W.R. Nelson, EGS3 electron gamma shower, SLAC report 210 (1978).

7) L. Bachman et al., A fine-grained electromagnetic lead-liquid scintillator calorimeter, Nucl. Instrum. Methods 206 (1983) 85 .

8) A. Drescher et al., The ARGUS electron-photon calorimeter, DESY 82-030 Report, May 1982. 


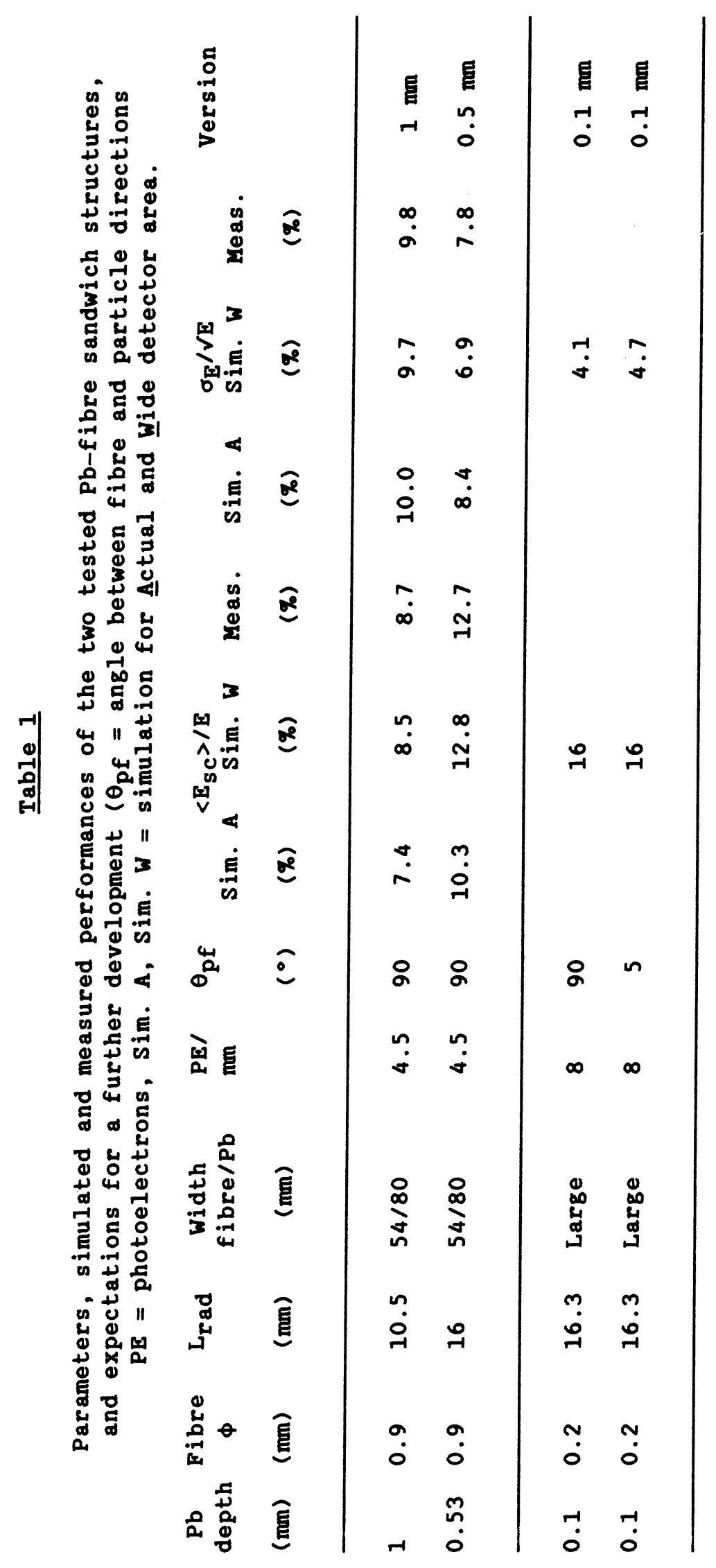




\section{Figure captions}

Fig. 1 : The structure of a test module.

Fig. 2 : Superposed distributions of the energy seen by the scintillators for $40 \mathrm{MeV}$ and $475 \mathrm{MeV}$ electrons and for $1 \mathrm{GeV}$ muons which were used to set the energy scale, in the $0.5 \mathrm{~mm} \mathrm{~Pb}$ plate version.

Fig. $3: E_{S c} / E$ and $\sigma_{E} / \sqrt{ }$ versus the electron energy $E$. Block (open) circles $=1 \mathrm{~mm}(0.5 \mathrm{~mm}) \mathrm{Pb}$ plates.

Fig. $4: \sigma_{E} / \sqrt{ }$ versus $\mathrm{Pb}$ sampling thickness. $\nabla$ Ge-Mi-Ne ${ }^{7}, \Delta$ ARGUS ${ }^{8}$ ) this work. Figures are the scintillator/PB volume ratios.a, EGS simulation for the above and for a $0.1 \mathrm{~mm}$ possible $\mathrm{Pb}-\mathrm{f}$ ibre sandwich. $\nabla$ EGS simulation for the laterally extended $0.5 \mathrm{~mm}$ version. 


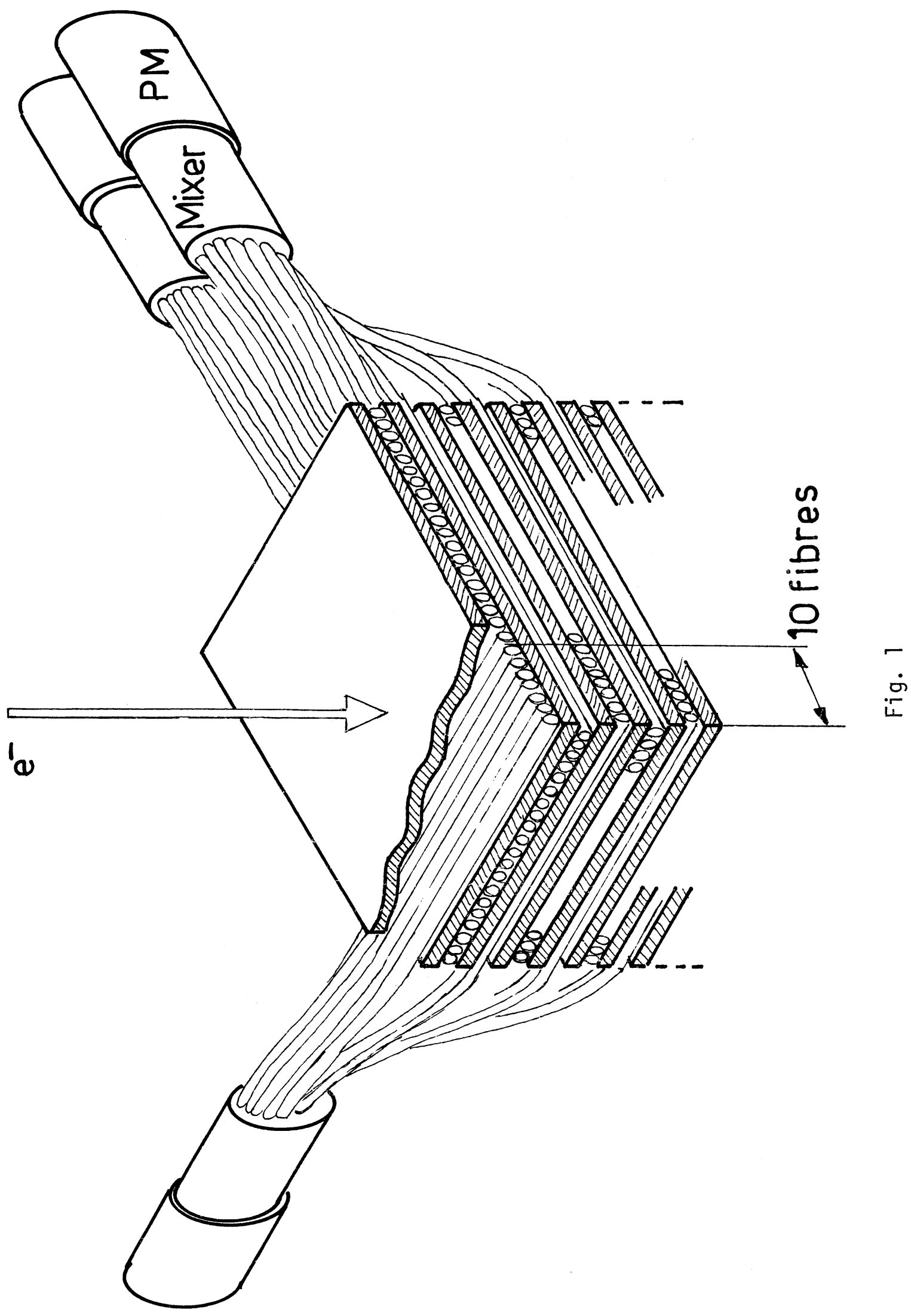




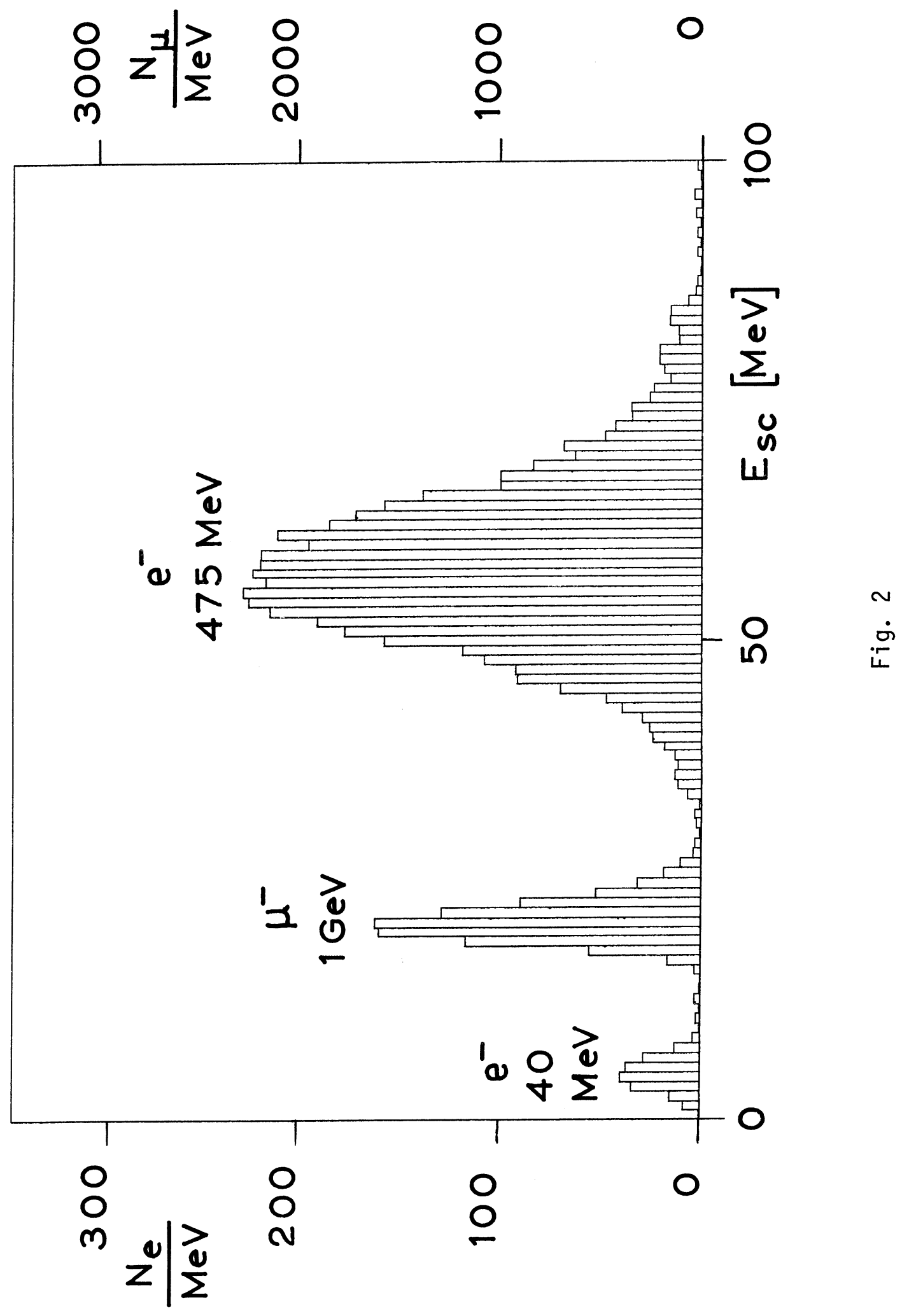




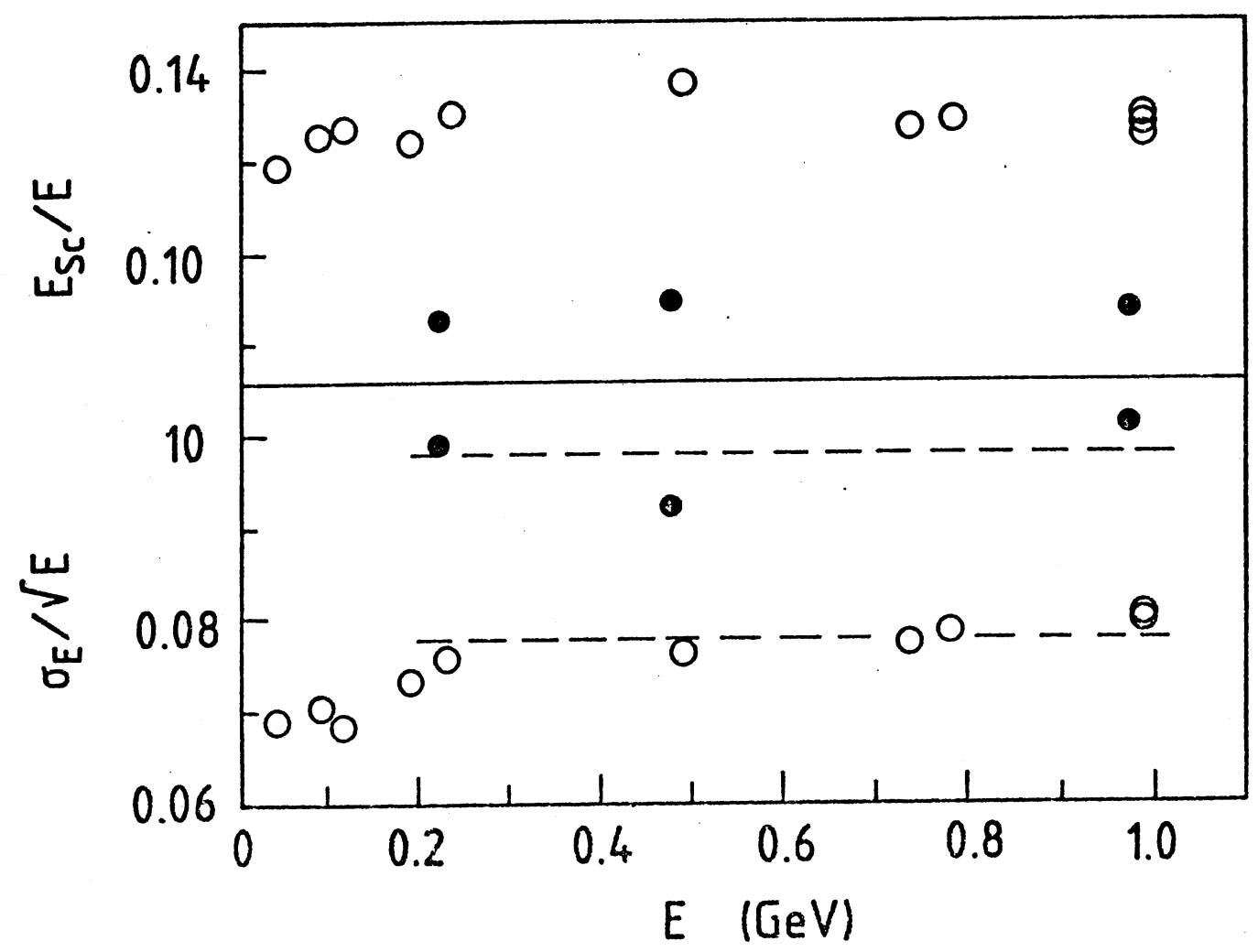

Fig. 3

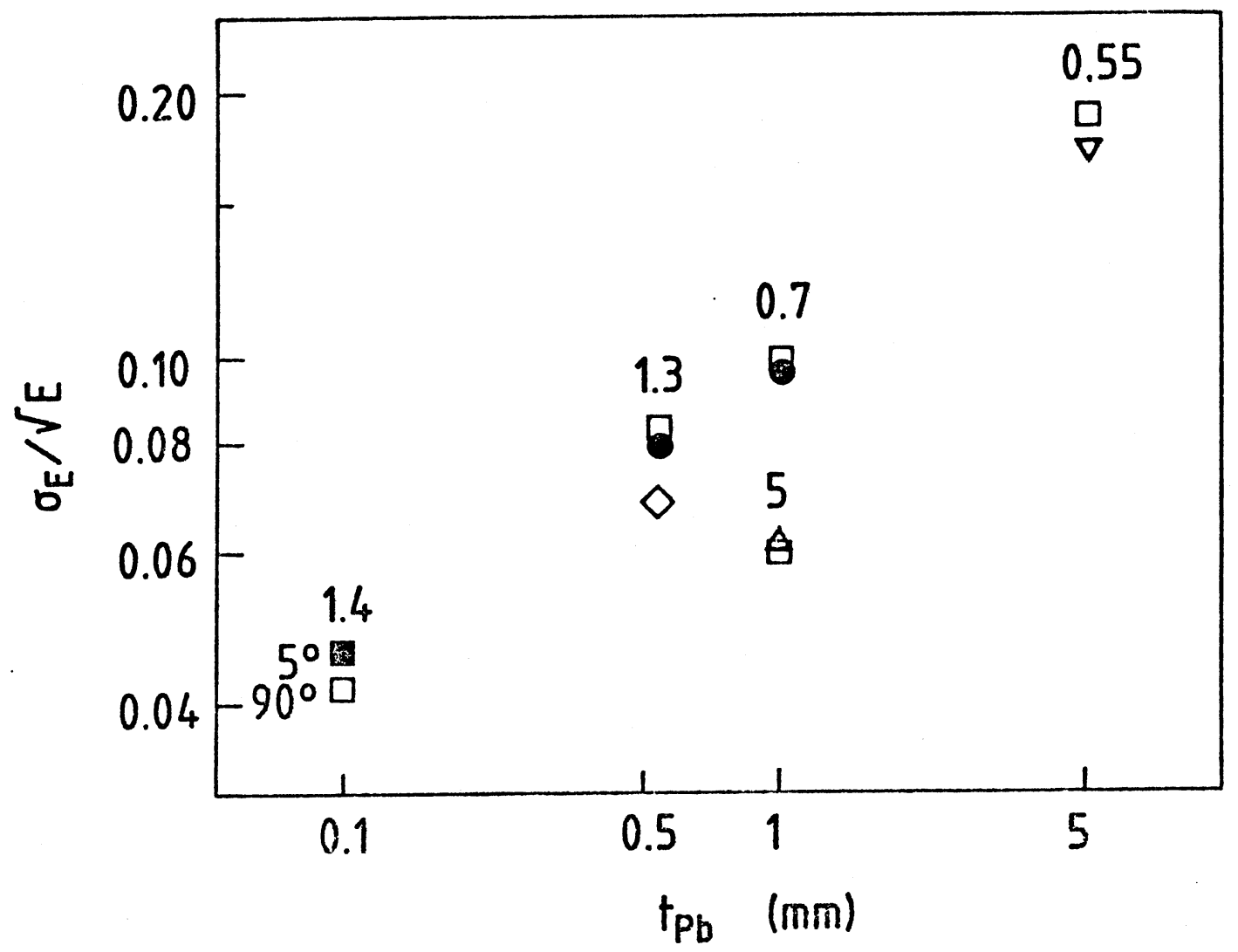

Fig. 4 